\title{
Biomimetic Sonar for Electrical Activation of the Auditory Pathway
}

\author{
D. Menniti, ${ }^{1}$ S. A. Pullano, ${ }^{1}$ M. G. Bianco, ${ }^{1}$ R. Citraro, ${ }^{2}$ E. Russo, ${ }^{2}$ \\ G. De Sarro, ${ }^{2}$ and A. S. Fiorillo ${ }^{1}$ \\ ${ }^{1}$ Chair of Biomedical Electronics, Department of Health Sciences, University "Magna Grocia” of Catanzaro, Catanzaro, Italy \\ ${ }^{2}$ Chair of Pharmacology, Department of Health Sciences, University "Magna Grocia" of Catanzaro, Catanzaro, Italy \\ Correspondence should be addressed to S. A. Pullano; pullano@unicz.it
}

Received 30 March 2017; Accepted 20 August 2017; Published 11 October 2017

Academic Editor: Stefania Campopiano

Copyright (C) 2017 D. Menniti et al. This is an open access article distributed under the Creative Commons Attribution License, which permits unrestricted use, distribution, and reproduction in any medium, provided the original work is properly cited.

\begin{abstract}
Relying on the mechanism of bat's echolocation system, a bioinspired electronic device has been developed to investigate the cortical activity of mammals in response to auditory sensorial stimuli. By means of implanted electrodes, acoustical information about the external environment generated by a biomimetic system and converted in electrical signals was delivered to anatomically selected structures of the auditory pathway. Electrocorticographic recordings showed that cerebral activity response is highly dependent on the information carried out by ultrasounds and is frequency-locked with the signal repetition rate. Frequency analysis reveals that delta and beta rhythm content increases, suggesting that sensorial information is successfully transferred and integrated. In addition, principal component analysis highlights how all the stimuli generate patterns of neural activity which can be clearly classified. The results show that brain response is modulated by echo signal features suggesting that spatial information sent by biomimetic sonar is efficiently interpreted and encoded by the auditory system. Consequently, these results give new perspective in artificial environmental perception, which could be used for developing new techniques useful in treating pathological conditions or influencing our perception of the surroundings.
\end{abstract}

\section{Background}

Ultrasound (US) plays an important role in the environmental perception of many species of mammals (such as rats and bats) for communication and survival; indeed, they use incoming US to gather information about incumbent danger, food availability, and navigational ranging [1]. Although Rodentia and Microchiroptera do not claim a common phylogenetic origin, recent literature reports that there are certain similarities in both mammals regarding anatomical organization $[2,3]$. Moreover, the cortical area arrangement and cortical frequency processing are similar to other mammals [4-6].

US frequency range of bats and rats partially overlaps and both eutherian lineages possess similar encoding process of mechanical US waves, although with different aims as spatial navigation and social communication $[7,8]$. In this study, a method for stimulating rats neural centres by using a bats bioinspired neuroelectronic interface is proposed. The electronic system for US signal processing is based on the natural sonar of Pteronotus parnellii and was previously investigated for modulating brain activity $[9,10]$. This study demonstrated ultrasound waves, which are properly decoded into bioelectrical signals, travelled along the acoustic pathway, and successfully reached cortical areas, processing sensorial information according to the environment or to cognitive demands. Similarly, exogenous stimulation may alter sensory perception by acting on the neural networks [11].

Hereafter, we have investigated how different signal patterns, emulating ultrasound echoes, affected brain activity by means of implanted electrodes connected to an electronic interface. Different patterns of echo signals at $50 \mathrm{kHz}$ were encoded into low-frequency signals (a few $\mathrm{Hz}$ ) according to auditory system transduction and codification processes, in order to bypass the auditory pathway and present the signal directly into the inferior colliculus (IC) of Wistar rats [12-14]. IC plays a strategic role in relaying and processing auditory information; indeed, receiving innervation from both the lateral lemniscus and the auditory cortex, the IC 
can be considered as an interface between the lower auditory pathway and the auditory cortex [15]. Moreover, the recent research on ascending auditory system suggests that the collicular projections influence the acoustic signal processing by modulating neural response $[16,17]$.

Electrocorticography (ECoG) recordings during stimulation were characterized by spike and spike-waves in accordance with the nature of audiogenic seizures. Cortical readings revealed that a frequency-lock condition exists, which had to do with the stimulus repetition rate, while the intensity of the stimulus changed neither the shape nor the frequency locking of the oscillations but only affected their amplitude. The relationship between US-parameters variation and the cortical activity showed the ability of the brain to process the different patterns generated by the sonar. By modulating neural rhythm, external stimulation could provide new perspectives for developing a stimulation technique for treating pathological conditions in which normal perception is altered or for directly conveying external information.

\section{Methods}

Microchiroptera send ultrasounds and receive echoes for orientation, as it happens in other vertebrates. Unlike other species such as rats, bats have extremely evolved echolocation system for orientation, prey detection, and capture, by adjusting echolocation calls (e.g., amplitude, time, and frequency), according to the specific situations. Along the auditory pathway, the frequency of the incoming signal is then expressed in a tonotopic organization, and amplitude is coded by varying the discharge rate of action potentials, while the interval between signals and the duration is coded by different temporal patterns of action potentials. Exploiting these information-bearing parameters (IBPs), different patterns of signals carrying information about the external environment have been used in order to verify whether natural oscillations in the frontal and parietal cortices can be influenced by cognitive activities in rats.

2.1. The Sonar System. The US sonar system is modeled on the echolocation mechanism of bats, which emit ultrasonic pulses and then listen to the returning echoes for orientation [18-20]. It is based on two hemicylindrical polyvinylidene fluoride (PVDF) transducers; one is used as transmitter of US signals and emulates the bat's vocal apparatus, while the other one is used as receiver and, together with the electronic interface, emulates the bat's auditory system.

The ultrasonic transducers are obtained by curving a strip of $40 \mu \mathrm{m}$ thick PVDF silver metallised on both large surfaces. They are structurally equal and interchangeable, with a resonance frequency which can be modified by changing the bending radius as described in [21]. The resonance frequency is described by the equation:

$$
f_{r}=\frac{1}{2 \pi r} \sqrt{\frac{Y}{\rho}},
$$

where $r$ denotes the curvature radius of PVDF film, $Y$ is Young's modulus, and $\rho$ is the polymer density. Blocking the two extremities of the piezoelectric film, the extensional motion is converted in the radial direction generating ultrasonic waves. Indeed, when the transmitter is excited by means of an electrical signal around its resonance frequency, the piezopolymer film is free to vibrate forward and backward transmitting an ultrasonic beam. The circuit for driving the transmitter consists of an operational power amplifier, a step-up transformer that increases signals from several Volt up to several hundred Volt $[18,19]$. The receiver converts the echo into an electrical signal. Before being transferred to the implanted microelectrodes, the receiver output is processed by a preamplifier with a high signal-to-noise ratio for increasing the signal intensity, and an active band-pass filter which amplifies the frequency components of interest, as normally happens on the basilar membrane. A full-wave precision rectifier and a low-pass filter are used to obtain a signal similar to the bioelectric patterns travelling along the acoustic nerve [19]. Figure 1 shows the electronic interface designed for processing the echo.

2.2. Signal Generation. In nature, emitted/received sounds analysis is of strategical importance for communication and survival among animal species. These sounds vary with time in several parameters including amplitude, frequency, and duration as well as the time interval occurring between each sounds. Also echolocating bats adjust their calls to optimize sonar performances. According to bat's echolocation system, by changing the IBPs, we investigated the effect induced by periodic electrical stimulation originated from US processed echoes on the cortical activity. Indeed, emulating the clicks emitted by bats, repetitive stimulus duration (SD) of $1 \mathrm{~ms}$ was transmitted, and the stimulation signal underwent progressive changes in terms of peak-to-peak amplitude which varied from 0.5 to $2 \mathrm{~V}$, interstimulus interval (ISI) which was varied from 50 to $500 \mathrm{~ms}$, and fundamental echo frequency fixed at 50 and $60 \mathrm{kHz}$ (see Figure 1) [20]. Intermittent stimulation has been used to mimic natural sonar behavior and to avoid adaptation in the IC. All the stimulation signals were constantly monitored by means of a digital oscilloscope during all stimulation phases. Ultrasonic echoes at $50 \mathrm{kHz}$ and $60 \mathrm{kHz}$ were chosen because all frequencies between 32 and $64 \mathrm{kHz}$ are naturally overrepresented in the acoustic fovea. This overrepresentation developed first in the cortex and later in the inferior colliculus suggesting a cortical origin and corticofugal influences [22]. Moreover, electrical stimuli were applied to IC whose response has shown a strong neurophysiological correlation with the echolocation calls [23].

2.3. Electrode Implantation. Five male Wistar rats (7 weeks old; Harlan Italy, Correzzana, Milan, Italy) were used in this study. The animals were kept under controlled environmental conditions $\left(2 \pm 2^{\circ} \mathrm{C} ; 60 \pm 5 \%\right.$ humidity; $12 \mathrm{~h} / 12 \mathrm{~h}$ light $/$ dark cycle; light on at 19.00). Access to food (standard laboratory chow) and tap water was ad lib until experimentation. The procedures involving animals and their care were conducted in conformity with international and national law and policies (EU Directive 2010/63/EU for animal experiments, ARRIVE guidelines, and the Basel declaration including the $3 \mathrm{R}$ concept). The experimental protocols and procedures 

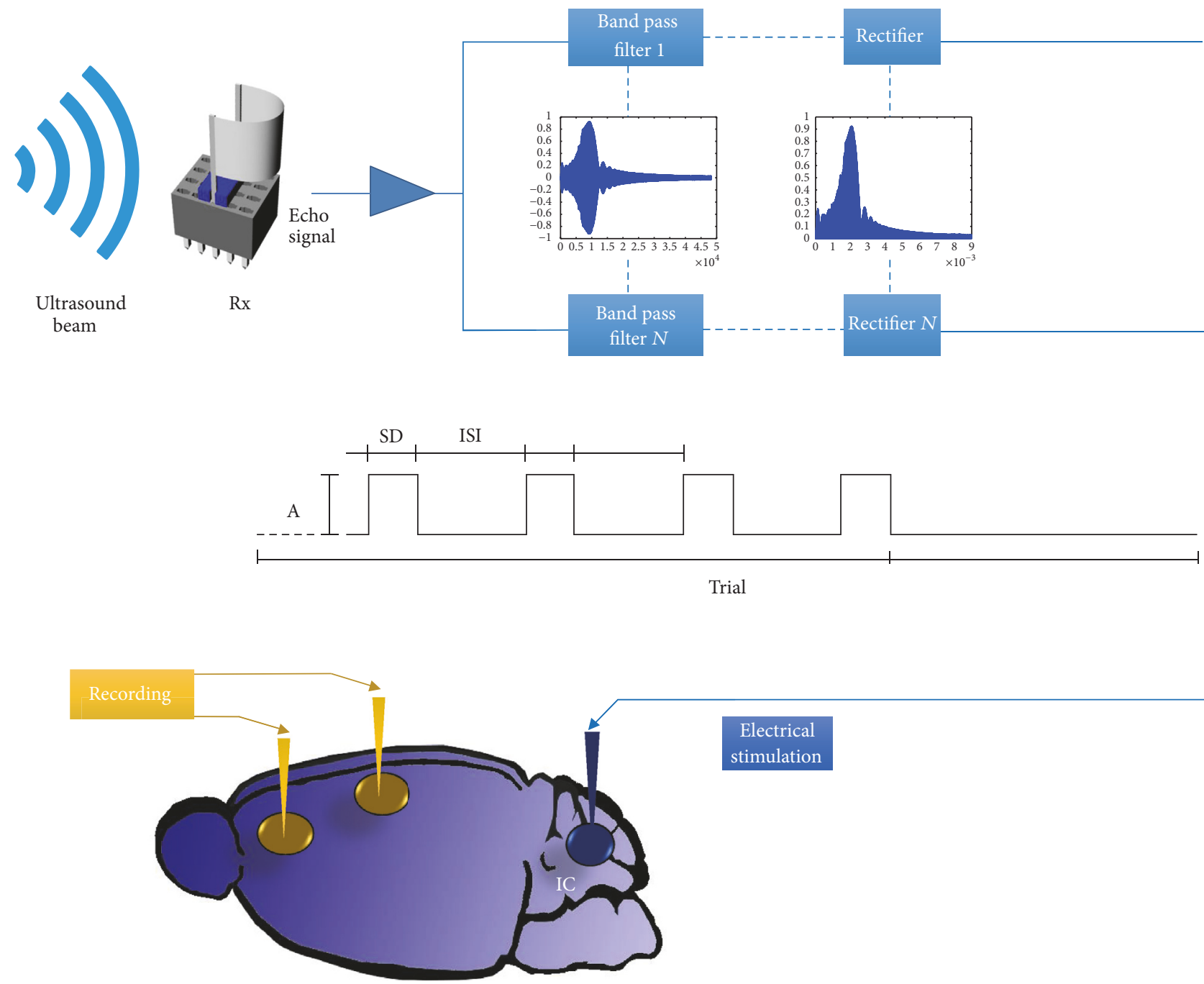

FIGURE 1: Block diagram of the electronic interface for echo processing and IC stimulation. The received echo is amplified band pass filtered and rectified in order to obtain unipolar signal similar to that generated in the cochlea. The signal is characterized by $1 \mathrm{~ms}$ SD with a variable ISI.

described in this manuscript were approved by the local ethical committee of the University of Catanzaro. All efforts were made to minimize animal suffering and to keep the number of animals used at a minimum. The electrodes used for stimulating and recording electrical activity in the brain consisted of a thin copper wire of 7-8 $\mathrm{mm}$ in length with a diameter of $10 \mu \mathrm{m}$. The wires were electrically insulated except for the tip. Each animal was anaesthetized through the administration of a mixture of tiletamine/zolazepam (1:1; Zoletil $100^{\circledR} ; 50 \mathrm{mg} / \mathrm{kg}$ i.p.; VIRBAC Srl, Milan, Italy) and subsequently inserted into a stereotaxic apparatus in order to carry out an accurate craniotomy which, through small holes in the skull, allowed the surgical implantation of two intracranial electrodes in the dura mater, immediately over the cortex, for the electrocorticographic recordings and one electrode in the inferior colliculus for stimulation. Electrocorticography with a noncephalic point of reference was used to detect brain activity: one electrode was positioned in the frontal cortex
$(\mathrm{AP}=-2 ; L=+2.5)$ and one in the parietal cortex (AP $=$ -6 ; $L=+2.5$ ) according to the atlas coordinates of Paxinos and Watson and a ground electrode was placed on the nasal bone. Cranioplastic cement anchored the electrodes to the skull [24]. For stimulation, these rats were implanted with one electrode in the inferior colliculus ( $\mathrm{AP}=0.7 ; L=+2 ; H=3.5$ ) according to the atlas coordinates of Paxinos and Watson. The wires were sufficiently spaced to avoid capacitive coupling between them. The animals were allowed at least 1 week of recovery and handled twice a day. The stimulating electrode was connected to the electronic interface and the recording electrodes to the ECoG acquisition system. ECoG were acquired using Stellate Harmonie system (Montreal, Quebec, Canada) connected to the animals by a flexible cable allowing the animals to move freely without twisting the cable.

2.4. Data Analysis. To assess how a complex neural network, like brain, codes and processes information, the ECoGs 
were recorded in absence of and during transcranial electrical stimulation [25]. The data were exported from the Stellate Harmonie system into ASCII text files that were then imported to MATLAB for analysis. A $50 \mathrm{~Hz}$ notch filter has been applied to remove electrical interferences [26]. Artifact removal from physiological signals such as eye blinks, muscular movements, and cardiac pulses in ECoG recordings was performed with Wavelet Transform (WT) through the use of discrete wavelet (DW) decomposition and thresholding method. DW transform decomposed signals in subbands recursively applying high-pass and low-pass filtering of the time domain using Daubechies- 8 mother function $[27,28]$. Parameters of similarity between the noised signal and the wavelet basis function are given by the output coefficients of both filters called detail $(D[n])$ and approximation $(A[n])$ coefficient at each level, respectively. Universal threshold implemented for wavelet coefficients shrinking was

$$
\begin{aligned}
K & =\sqrt{2 \log N} \sigma, \\
\sigma^{2} & =\operatorname{median}\left(\frac{\left|C_{a}\right|}{0.6745}\right),
\end{aligned}
$$

where " $K$ " is the threshold value, " $N$ " is the length of the ECoG signal, and " $C_{a}$ " is the wavelet coefficients at " $a$ " level of decomposition.

ECoG power spectrum density (PSD) was acquired in the range $0 \div 100 \mathrm{~Hz}$. The relationship between periodic stimulation and ECoG activity was analyzed with the Fast Fourier Transform (FFT) besides comparison between a "prestimulus" baseline and the ECoG under the different experimental conditions. In fact, some important features of the electrophysiological signals determined by timefrequency analysis (e.g., different brain states, from sleep to cognitive processing) are reflected by different oscillations in the ECoG [27]. Spectral analysis was carried out within each band of interest, that is, delta $(1-4 \mathrm{~Hz})$, theta $(4-7 \mathrm{~Hz})$, alpha $(8-13 \mathrm{~Hz})$, beta $(14-29 \mathrm{~Hz})$, and gamma $(30-100 \mathrm{~Hz})$.

To assess dynamic changes in spectral power over time we used Chronux toolbox which computes a spectrogram based on a multitaper analysis. The spectrum was computed over a sliding $50 \mathrm{~ms}$ window. The $y$-axis represents frequency in $\mathrm{Hz}$ and the $x$-axis represents time. Power at a given frequency was indicated by the color of each point. Hot colors represent higher energy and cooler colors represent lower energy.

Principal component analysis (PCA) is one of the most common methods for analyzing large data sets, reducing the dimensionality, allowing the extraction of data features. Classification of ECoG signals plays an important role in many neurological studies, for example, for staging a neurologic disease or for brain-machine interface applications $[29,30]$. In this study, PCA was applied to ECoG, for highlighting how rats' brain codes different stimulus features [31]. We began by binning the data in a set of vectors containing processed ECoG epochs. From these vectors, covariance matrices were constructed.

\section{Results}

During stimulation, the recorded activity was clearly distinguishable from background activity. Indeed, the cortical responses recorded by ECoG from both frontal and parietal electrodes have shown a correlation with the features of the stimuli, such as amplitude and ISI. By adjusting the amplitude of the electrical stimulation, we observed changes in activity on the parietal lobe as shown in Figure 2. Varying the US echo frequency, cortical activity did not change significantly.

ISI variations induced in ECoG shape a transition from spike-waves to spike components when the interval time between trains was varied from 50 to $500 \mathrm{~ms}$, as shown in Figure 3. Moreover, cortical readings indicate that a frequency-lock condition exists, which has to do with the stimulus repetition rate.

ECoGs acquired in the parietal region during stimulation, at different amplitudes, showed that the more the stimulus intensity was decreased, the longer the latency response was. Following stimulation, the neuronal activity returned to its normal background pattern without any latency time. However, ECoG normalization was also exhibited during stimulation, which is attributable to chemical inhibition of the synaptic transmission, which could be the result of neurotransmitters depletion as well as the presence of inhibitory neurotransmitters released by the cerebral cortex.

Power spectral density estimation on the parietal and frontal cortex recordings highlighted that a higher cortical response in the beta rhythm was induced if compared with background activity. The higher contents of electrical oscillations in this band were not related to variations in the amplitude (Figure 4) and the ISI (Figure 5) of the incoming information. On the contrary, delta oscillations increased only during stimulation at 50 and $100 \mathrm{~ms}$ since the signal frequency was in the range $1-4 \mathrm{~Hz}$ (Figure 5). Other frequencybands were not subject to any significant changes.

The dynamic analysis of ECoG signals, performed during the transition between the baseline and the stimulation state, confirmed a change in the spectral energy content following the auditory stimulation (Figure 6).

PCA is used to detect stimulus-related features of the dataset more precisely by reducing the data variability. PCA performed on the ECoG recorded from the parietal (Figures 7 and 8) and frontal cortex (Figures 9 and 10), when variations of the amplitude and ISI values were carried out, confirmed that stimulation elicited an alteration in the ECoG allowing a classification of the ongoing cortical activity recorded during different stimulation conditions. The distribution of ECoG epochs detected by the parietal electrode, when the stimulus parameters vary, has shown more accurate classification performances than the one generated by the frontal channel.

\section{Discussion}

The analysis of data showed that neurons modulated their functional state in response to the external stimulus and its features, strongly suggesting ECoG reactivity to electrical inputs. The parietal lobe was considered the focal point of our investigation since it is highly sensitive to acoustic stimuli 


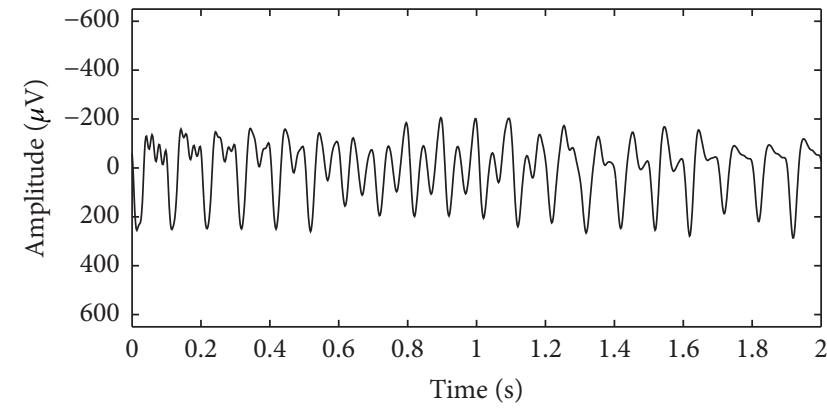

(a)

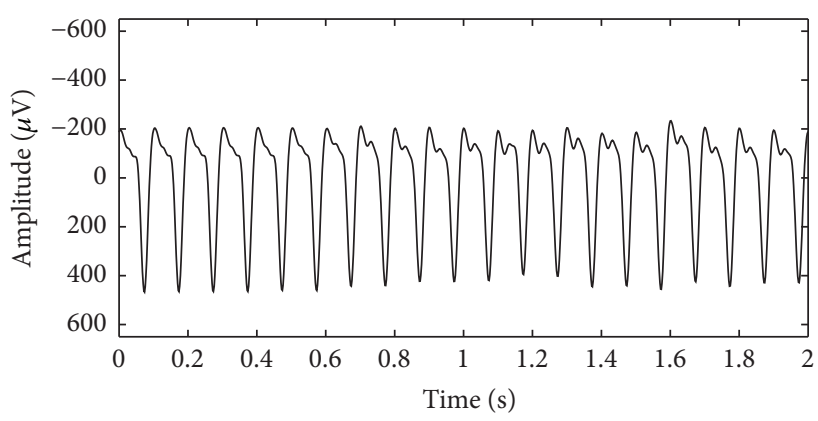

(c)

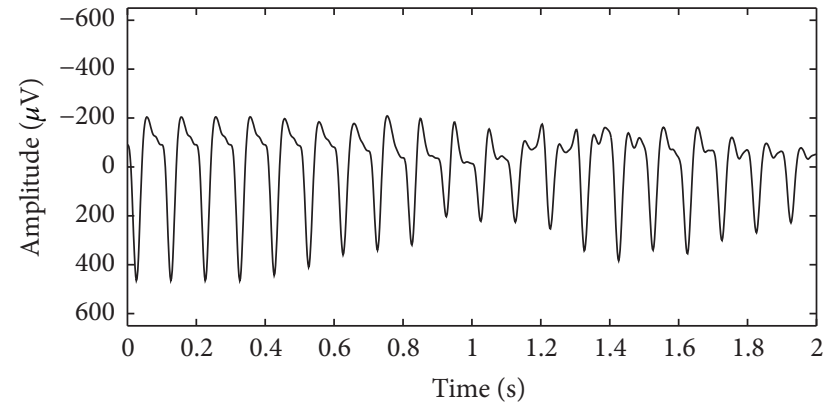

(b)

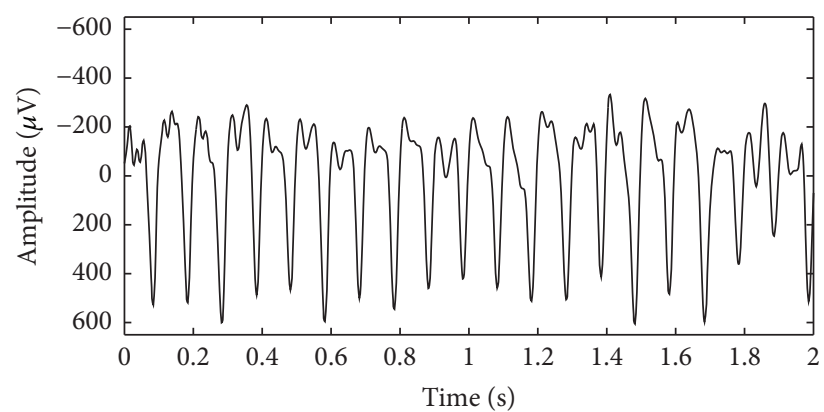

(d)

FIGURE 2: ECoG recorded from the parietal cortex characterized by spike and spike-waves discharges influenced by the stimulus intensity set at $0.5 \mathrm{~V}(\mathrm{a}), 1 \mathrm{~V}(\mathrm{~b}), 1.5 \mathrm{~V}(\mathrm{c})$, and $2 \mathrm{~V}(\mathrm{~d})$.

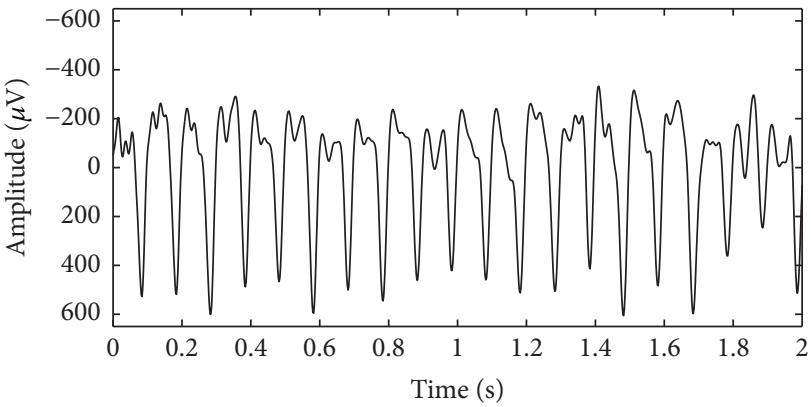

(a)

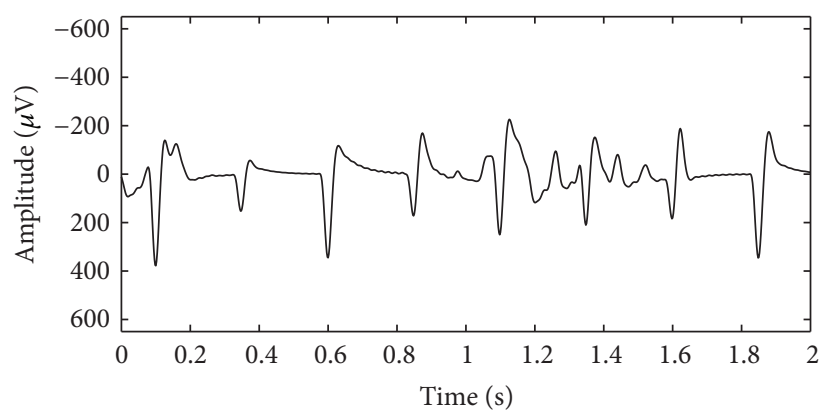

(c)

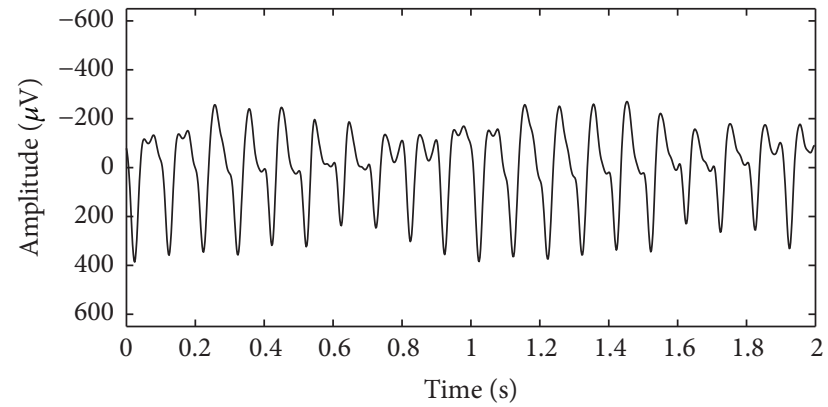

(b)

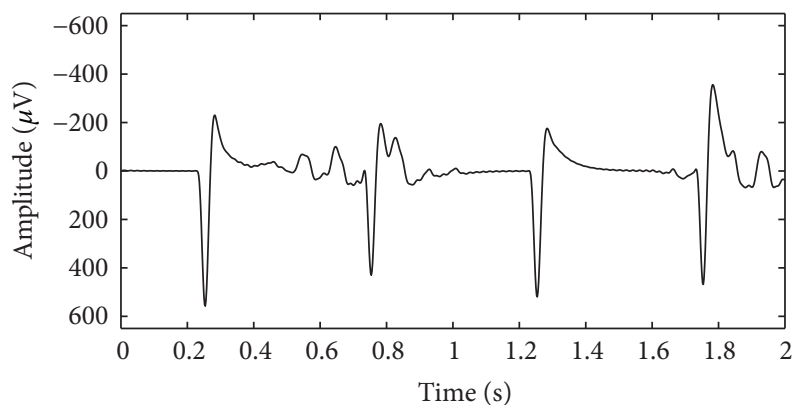

(d)

FIGURE 3: ECoG activity recorded from the parietal cortex showing a transition from spike-waves when the ISI is set to $50 \mathrm{~ms}$ (a) and $100 \mathrm{~ms}$ (b) to spike components when the ISI is set at $250 \mathrm{~ms}$ (c) and $500 \mathrm{~ms}$ (d). 


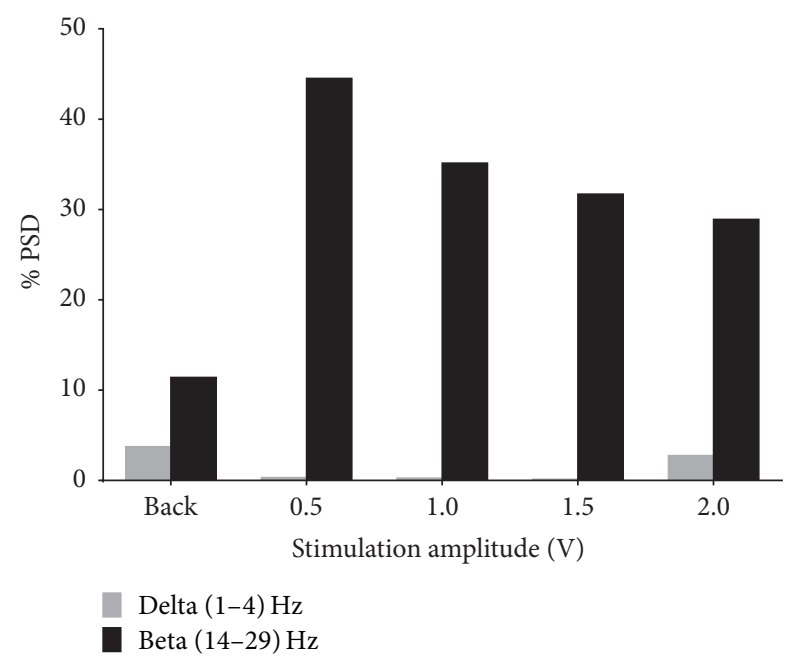

FIGURE 4: PSD evaluated in the delta and beta frequency band stimulating the IC at variable amplitude.

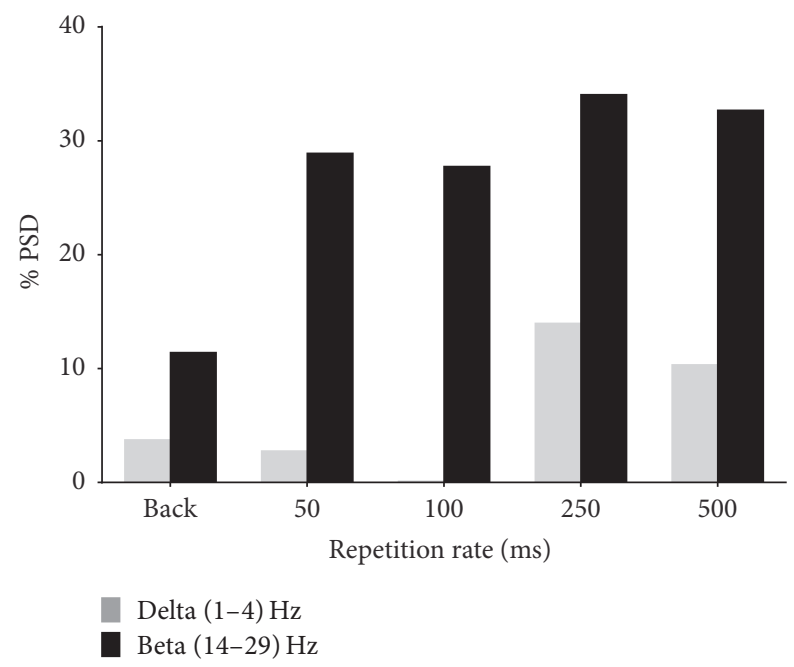

FIGURE 5: PSD evaluated in the delta and beta frequency band stimulating the IC with variable ISI.

and plays an important role in integrating information from different sensory systems to build an image of the world. Our aim was to analyze the effects of different stimulation patterns on ECoG activity both in time and in frequency domain.

As the stimulation intensity decreased, the cortical activity attenuated accordingly, while variations in the ISI values were not always directly correlated to changes in the ECoG amplitude; therefore, the relationship between repetition rate and strength of brain activity was not significant. However, the frequency and the shape of neural response were correlated to the repetition rate. Indeed, the brain adapted to slow auditory stimuli with bursts of spikes at lower frequency instead of spike-waves occurring at faster frequency.

The oscillations evoked in the beta range revealed that the brain was able to process the sensory information delivered into the neural network by means of ultrasound pulses. In fact, beta oscillations in response to repeated upcoming

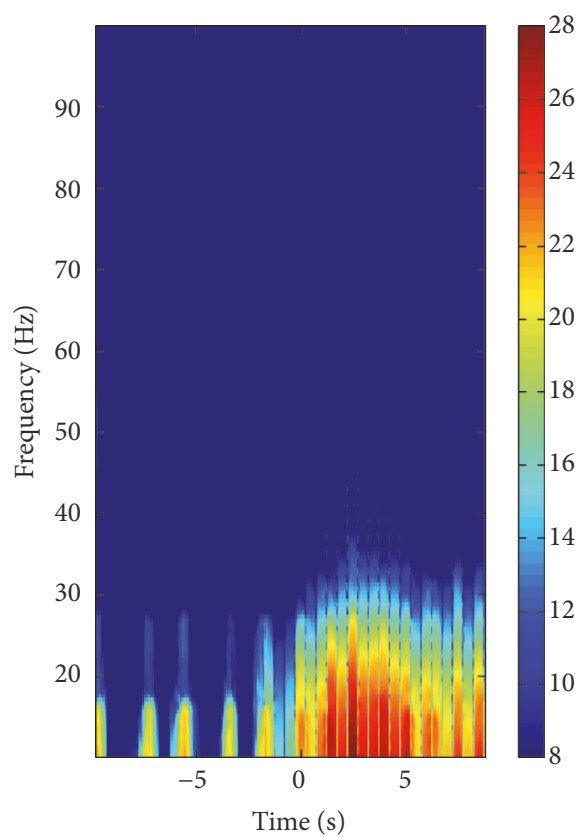

FIGURE 6: Spectrogram of neural response before and during intermittent US stimulation delivered at time $t=0$.

auditory stimuli and a relationship between elevated beta activity and immobilization during transcranial current stimulation at $20 \mathrm{~Hz}$ have already been observed [32,33]. Timefrequency power plot, further, demonstrated the difference between baseline power and power in response to stimulation. The onset of the stimulus induced spectral perturbations that corresponded to the transient synchronization of neuronal populations to the stimulus.

Spikes and spike-waves detection, clearly different from spontaneous brain activity, confirmed the influence on cortical activity of the stimuli. We pursued this matter observing the relationship between the frequency of the external signal, denoted as $X$, and that of the evoked response, denoted as $Y$. When the repetition rate was $50 \mathrm{~ms}$ and the amplitude of the stimulation was varied between 0.5 and $2 \mathrm{~V}$, brain oscillations were frequency-locked to the frequency of the stimulus in a ratio of $2: 1$.

This probably occurred because the induced rhythm required synaptic kinetics which were too fast for the correct neurological transfer of information. At $100 \mathrm{~ms}$, we observed a 1:1 frequency ratio, proving that the spike-waves were perfectly locked to the external stimuli at $2 \mathrm{~V}$. This condition remained unchanged when the electrical pulses were delivered at repetition rate of 250 and $500 \mathrm{~ms}$. By changing the frequency of the waves from 50 to $60 \mathrm{kHz}$, the neuronal activity gave rise to a $2: 1 \mathrm{ECoG}$ frequency-lock because the repetition rate held steady at $50 \mathrm{~ms}$. Analysis of spikes and spike-waves highlighted how epileptic events produced by the neural network were frequency-locked to the intermittent external stimulus at a ratio of $X: Y$, depending on the repetition rate.

To validate and clarify the stimulation patterns on ECoG activity we performed PCA in both frontal and parietal cortex. PCA from parietal channel has shown how all the stimuli generated patterns of neural activity clearly separated, while 


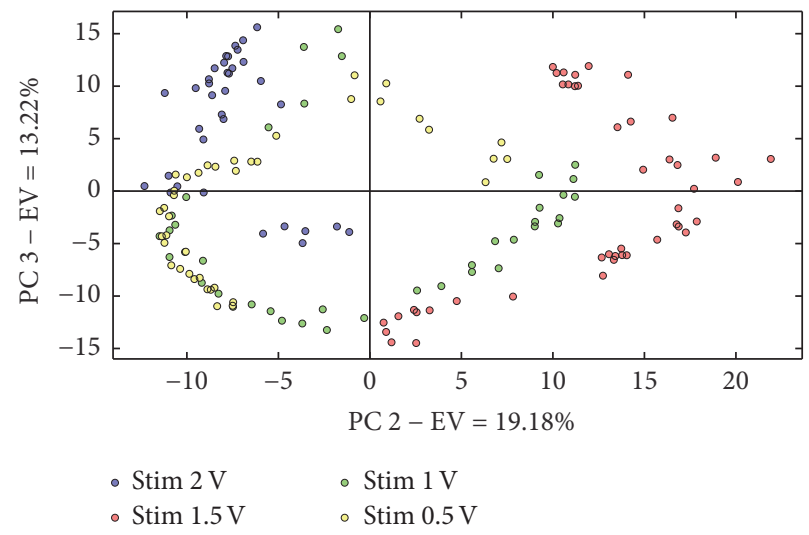

FIgURE 7: PCA score plot evaluated from ECoG recordings in the parietal cortex at variable amplitude.

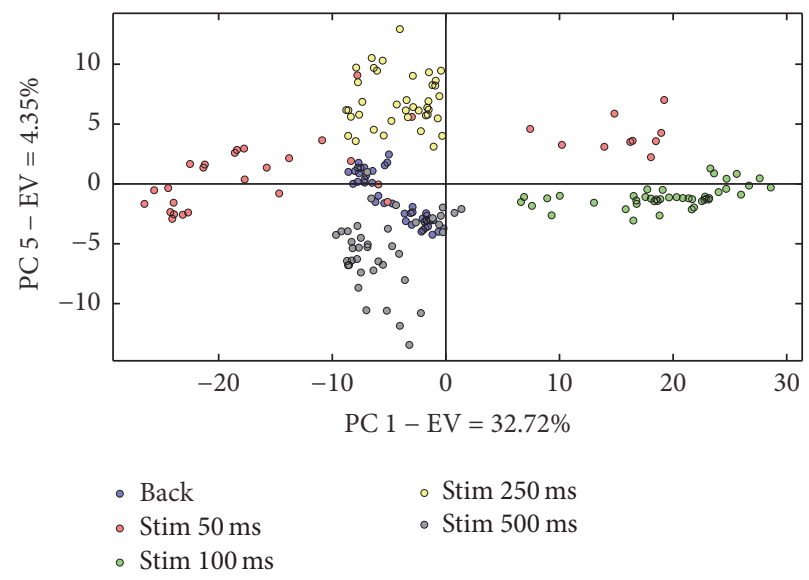

FIGURE 8: PCA score plot evaluated from ECoG recordings in the parietal cortex at variable ISI.

PCA applied to the other channel suggested that stimulusevoked activity was attenuated in frontal area since spatiotemporal information mainly activated the parietal region as reported in literature [34]. The 2D score plot in Figure 7 contains the distribution of cortical activity detected by the parietal electrode in response to different amplitude values of the US stimuli: at $0.5 \mathrm{~V} \mathrm{ECoG}$ recording is localized in the first, second, and third quadrants, at $1 \mathrm{~V}$ it is mainly concentrated in the third and fourth quadrants, and at $1.5 \mathrm{~V}$ it is placed in the first and fourth quadrants, while at $2 \mathrm{~V}$ it is enclosed in the second quadrant. Figure 8 shows the score plot generated by ECoG recorded at different values of the repetition rate of the stimulation signal: at $50 \mathrm{~ms}$ brain response is scattered into the first, the second, and the third quadrants, at $100 \mathrm{~ms}$ it mainly occupies the fourth quadrant, and at $250 \mathrm{~ms}$ it is grouped in a small area in the second quadrant, while at $500 \mathrm{~ms}$ it is principally concentrated in the third quadrant. Moreover, background activity falls between the third and the second quadrants.

Figure 9 displays the biplot of the first two components of a PCA generated by plotting the scores of the data set obtained from the ECoG recorded by the frontal electrode when the amplitude of the US stimuli changes: at $0.5 \mathrm{~V} \mathrm{ECoG}$ recording is mainly located in the right upper side of the third quadrant, at $1 \mathrm{~V}$ it has scores in the first, second, and fourth quadrants, and at $1.5 \mathrm{~V}$ it lies in the right upper quadrant, while at $2 \mathrm{~V}$ it is spread out between the second and the fourth quadrants. Figure 10 shows the score plot calculated from the ECoG recorded from the frontal electrode by setting different values for the time between pulses: at $50 \mathrm{~ms}$ neural activity is represented by a large dispersion of points in the plot, at $100 \mathrm{~ms}$ it creates a cluster of points very close to each other and concentrated in the third quadrant, and at $250 \mathrm{~ms}$ and at $500 \mathrm{~ms}$ brain responses overlap in the plot.

The results show how different features of the external stimulation affect epileptic oscillations in terms of frequency synchronization, amplitude, and shape on the ECoG traces. All this provides evidence of signal-related brain activity and suggests that when the inferior colliculus is externally stimulated and the sensation is perceived, and the cortical response is specific for every signal sent, making this stimulation technique interesting and suitable for carrying information. Moreover, periodic pulse stimulation leads to frequency locking and thus can be of help in understanding epileptic seizure control through the modulation of the activity of the brain $[35,36]$. 


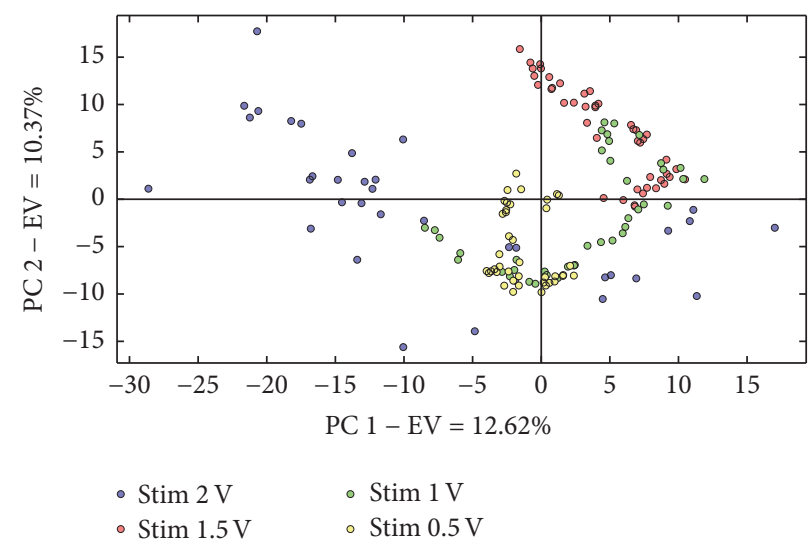

FIGURE 9: PCA score plot evaluated from ECoG recordings in the frontal cortex at variable amplitude.

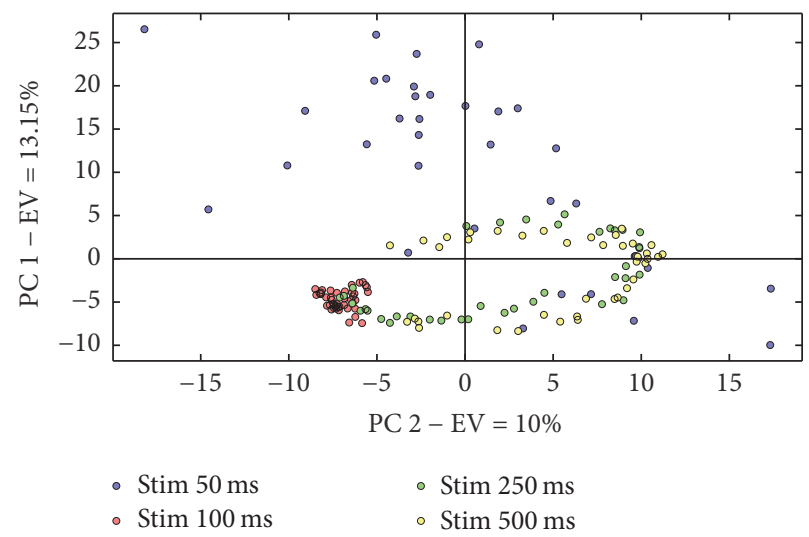

FIgURE 10: PCA score plot evaluated from ECoG recordings in the frontal cortex at variable ISI.

\section{Conclusion}

In this study, we stimulated the acoustic pathway with pseudo-action potentials generated by bioinspired electronic interface. The stimulation parameters, in particular amplitude and echo frequency, were each significantly correlated to changes in neuronal response. We demonstrated that ultrasonic waves, properly processed and delivered into the inferior colliculus, by means of implanted electrodes, gave rise to a brain response modulated by echo signal features suggesting that spatial information sent by our stimulation signal was efficiently interpreted and encoded by the auditory system. The electronic interface developed could be a promising strategy for providing environmental information bypassing the peripheral auditory system. We found that IC stimulation brought to a frequency-locking condition within a certain range and that could have an impact for the development of new methods for the control of pathological brain activity although further researches are necessary to develop a device, based on our technology, which can efficaciously act on the cognitive and nervous systems.

\section{Disclosure}

D. Menniti and S. A. Pullano are co-first authors.

\section{Conflicts of Interest}

The authors declare that they have no conflicts of interest.

\section{Authors' Contributions}

D. Menniti and S. A. Pullano contributed equally to this work.

\section{Acknowledgments}

The authors would like to thank Eng. Francesco Ruberto and Dr. Nicola Costa for technical support.

\section{References}

[1] F. O. Walker, M. S. Cartwright, E. R. Wiesler, and J. Caress, "Ultrasound of nerve and muscle," Clinical Neurophysiology, vol. 115, no. 3, pp. 495-507, 2004.

[2] W. J. Murphy, E. Eizirik, W. E. Johnson, Y. P. Zhang, O. A. Ryder, and S. J. O'Brien, "Molecular phylogenetics and the origins of placental mammals," Nature, vol. 409, no. 6820, pp. 614-618, 2001.

[3] E. H. Buhl and J. F. Dann, "Cytoarchitecture, neuronal composition, and entorhinal afferents of the flying fox hippocampus," Hippocampus, vol. 1, no. 2, pp. 131-152, 1991. 
[4] M. Fyhn, S. Molden, M. P. Witter, E. I. Moser, and M.-B. Moser, "Spatial representation in the entorhinal cortex," Science, vol. 305, no. 5688, pp. 1258-1264, 2004.

[5] M. M. Yartsev, M. P. Witter, and N. Ulanovsky, "Grid cells without theta oscillations in the entorhinal cortex of bats," Nature, vol. 479, no. 7371, pp. 103-107, 2011.

[6] J. G. Heys, K. M. MacLeod, C. F. Moss, and M. E. Hasselmo, "Bat and rat neurons differ in theta-frequency resonance despite similar coding of space," Science, vol. 340, no. 6130, pp. 363-367, 2013.

[7] A. J. Parsana, N. Li, and T. H. Brown, "Positive and negative ultrasonic social signals elicit opposing firing patterns in rat amygdala," Behavioural Brain Research, vol. 226, no. 1, pp. 7786, 2012.

[8] H.-U. Schnitzler, C. F. Moss, and A. Denzinger, "From spatial orientation to food acquisition in echolocating bats," Trends in Ecology and Evolution, vol. 18, no. 8, pp. 386-394, 2003.

[9] J. A. Butman and N. Suga, "Synaptic mechanisms shaping delay-tuned combination-sensitivity in the auditory thalamus of mustached bats," Hearing Research, vol. 331, pp. 69-82, 2016.

[10] M. Kössl, J. Hechavarria, C. Voss, M. Schaefer, and M. Vater, "Bat auditory cortex - model for general mammalian auditory computation or special design solution for active time perception?" European Journal of Neuroscience, vol. 41, no. 5, pp. 518$532,2015$.

[11] L. N. Spezia Adachi, A. S. Quevedo, A. de Souza et al., "Exogenously induced brain activation regulates neuronal activity by top-down modulation: conceptualized model for electrical brain stimulation," Experimental Brain Research, vol. 233, no. 5, pp. 1377-1389, 2015.

[12] A. S. Fiorillo, S. A. Pullano, C. D. Critello, R. Citraro, G. De Sarro, and E. Russo, "Measurements of rat brain activity originating from ultrasound waves in air," in Proceedings of the IEEE International Symposium on Medical Measurements and Applications, MeMeA 2013, pp. 233-236, Gatineau, Canada, March 2013.

[13] M. Bianco, S. Pullano, D. Menniti, A. Fiorillo, and E. de VillersSidani, "Evaluation of auditory cortex tonotopical organization in rats Through Principal Component Analysis," in Proceedings of the 2017 IEEE International Symposium on Medical Measurements and Applications (MeMeA), pp. 222-226, Rochester, MN, USA, May 2017.

[14] D. Menniti, M. G. Bianco, S. A. Pullano et al., "Activation of bottom-up and top-down auditory pathways by US sensors based interface," in Proceedings of the 2017 7th IEEE International Workshop on Advances in Sensors and Interfaces (IWASI), pp. 15-16, Vieste, Italy, June 2017.

[15] M. S. Malmierca, "The inferior colliculus: A center for convergence of ascending and descending auditory information," Neuroembryology and Aging, vol. 3, no. 4, pp. 215-229, 2004.

[16] V. M. Bajo and A. J. King, "Cortical modulation of auditory processing in the midbrain," Frontiers in Neural Circuits, vol. 6, article 114, 2012.

[17] Y. Li, M. S. Evans, and C. L. Faingold, "Synaptic response patterns of neurons in the cortex of rat inferior colliculus," Hearing Research, vol. 137, no. 1-2, pp. 15-28, 1999.

[18] A. S. Fiorillo and S. A. Pullano, "Ferroelectric polymer for bio-sonar replica," in Ferroelectrics, L. Mickaël, Ed., pp. 75-94, Intech, Rijeka, Croatia, 2011.
[19] A. S. Fiorillo and G. D'Angelo, "Echo signals processing with neural network in bat-like sonars based on PVDF," in Proceedings of the 2002 IEEE Ultrasonics Symposium, Munich, Germany, 2009.

[20] N. Suga, "Cortical computational maps for auditory imaging," Neural Networks, vol. 3, no. 1, pp. 3-21, 1990.

[21] A. S. Fiorillo, "Design and characterization of a PVDF ultrasonic range sensor," IEEE Transactions on Ultrasonics, Ferroelectrics, and Frequency Control, vol. 39, no. 6, pp. 688-692, 1992.

[22] H. Kim and S. Bao, "Experience-dependent overrepresentation of ultrasonic vocalization frequencies in the rat primary auditory cortex," Journal of Neurophysiology, vol. 110, no. 5, pp. 10871096, 2013.

[23] X. F. Luo, J. Ma, A. A. Li, F. J. Wu, Q. C. Chen, and S. Y. Zhang, "Echolocation Calls and Neurophysiological Correlations with Auditory Response Properties in the Inferior Colliculus of Pipistrellus abramus," Zoological Studies, vol. 46, pp. 622-630, 2007.

[24] G. Paxinos and C. Watson, The Rat Brain in Stereotaxic Coordinates, vol. 4th, 1998.

[25] P. Gryboś, P. Kmon, M. Zołqdź et al., "64 channel neural recording amplifier with tunable bandwidth in $180 \mathrm{~nm}$ CMOS technology," Metrology and Measurement Systems, vol. 18, no. 4, pp. 631-644, 2011.

[26] Q. Huang, S. Chang, J. Peng, X. Mao, Y. Zhou, and H. Wang, "An implementation of SOPC-based neural monitoring system," IEEE Transactions on Instrumentation and Measurement, vol. 61, no. 9, pp. 2469-2475, 2012.

[27] N. Mammone, F. La Foresta, and F. C. Morabito, "Automatic artifact rejection from multichannel scalp EEG by wavelet ICA," IEEE Sensors Journal, vol. 12, no. 3, pp. 533-542, 2012.

[28] A. E Hramov, A. A. Koronovskii, V. A. Makarov, A. N. Pavlov, and E. Sitnikova, Wavelets in Neuroscience, Springer Series in Synergetics, Springer, Berlin, Germany, 2014.

[29] J. C. G. D. Costa, P. J. G. Da-Silva, R. M. V. R. Almeida, and A. F. C. Infantosi, "Validation in principal components analysis applied to EEG data," Computational and Mathematical Methods in Medicine, vol. 2014, Article ID 413801, 2014.

[30] N. A. Khan and S. Ali, "Classification of EEG signals using adaptive Time-Frequency distributions," Metrology and Measurement Systems, vol. 23, no. 2, pp. 251-260, 2016.

[31] J. K. Chapin and M. A. L. Nicolelis, "Principal component analysis of neuronal ensemble activity reveals multidimensional somatosensory representations," Journal of Neuroscience Methods, vol. 94, no. 1, pp. 121-140, 1999.

[32] C. Haenschel, T. Baldeweg, R. J. Croft, M. Whittington, and J. Gruzelier, "Gamma and beta frequency oscillations in response to novel auditory stimuli: A comparison of human electroencephalogram (EEG) data with in vitro models," Proceedings of the National Academy of Sciences of the United States of America, vol. 97, no. 13, pp. 7645-7650, 2000.

[33] A. Pogosyan, L. D. Gaynor, A. Eusebio, and P. Brown, "Boosting cortical activity at beta-band frequencies slows movement in humans," Current Biology, vol. 19, no. 19, pp. 1637-1641, 2009.

[34] H.-Y. Cho, K.-T. Kim, and J.-H. Jung, "Effects of neurofeedback and computer-assisted cognitive rehabilitation on relative brain wave ratios and activities of daily living of stroke patients: A randomized control trial," Journal of Physical Therapy Science, vol. 28, no. 7, pp. 2154-2158, 2016.

[35] R. Citraro, A. Leo, P. De Fazio, G. De Sarro, and E. Russo, "Antidepressants but not antipsychotics have antiepileptogenic 
effects with limited effects on comorbid depressive-like behaviour in the WAG/Rij rat model of absence epilepsy," British Journal of Pharmacology, vol. 172, no. 12, pp. 3177-3188, 2015.

[36] E. Russo, F. Scicchitano, R. Citraro et al., "Protective activity of $\alpha$-lactoalbumin (ALAC), a whey protein rich in tryptophan, in rodent models of epileptogenesis," Neuroscience, vol. 226, pp. 282-288, 2012. 


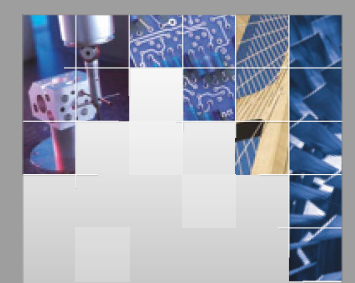

\section{Enfincering}
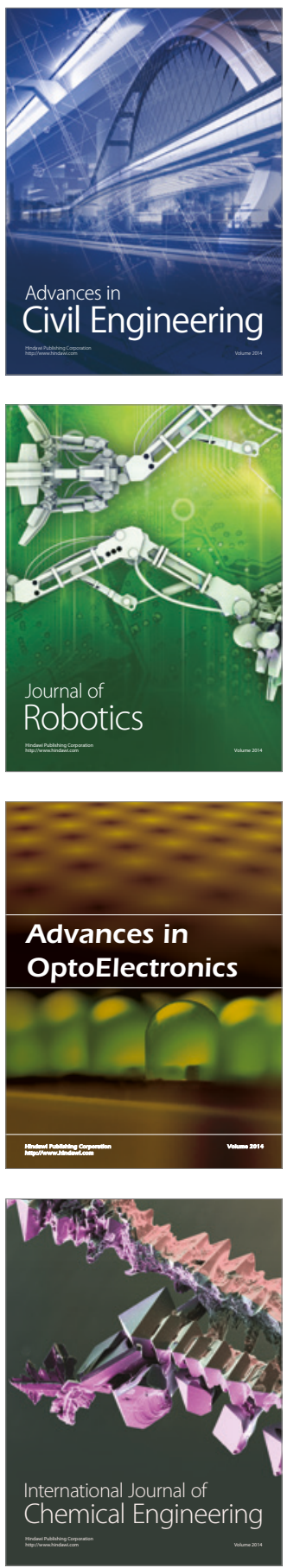

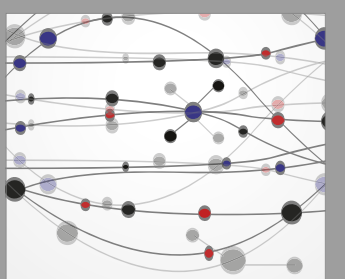

The Scientific World Journal

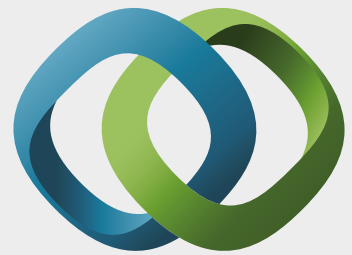

\section{Hindawi}

Submit your manuscripts at

https://www.hindawi.com
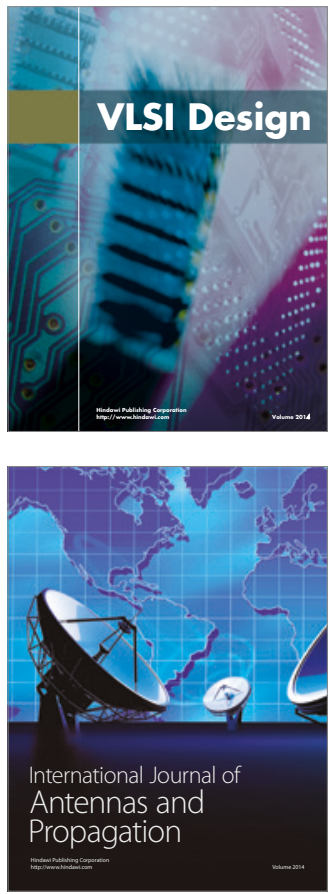

\section{Rotating}

Machinery
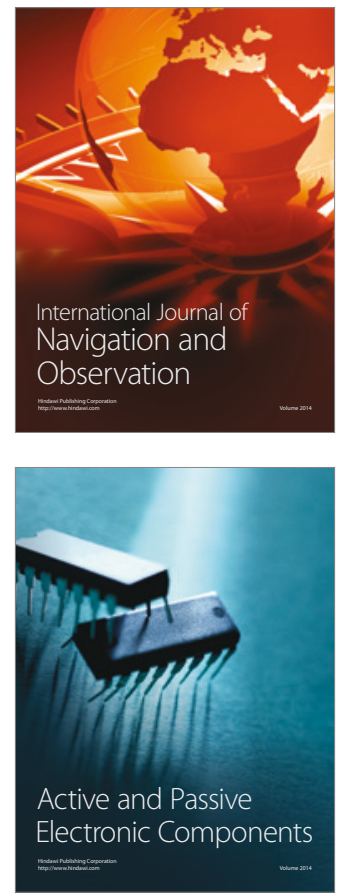
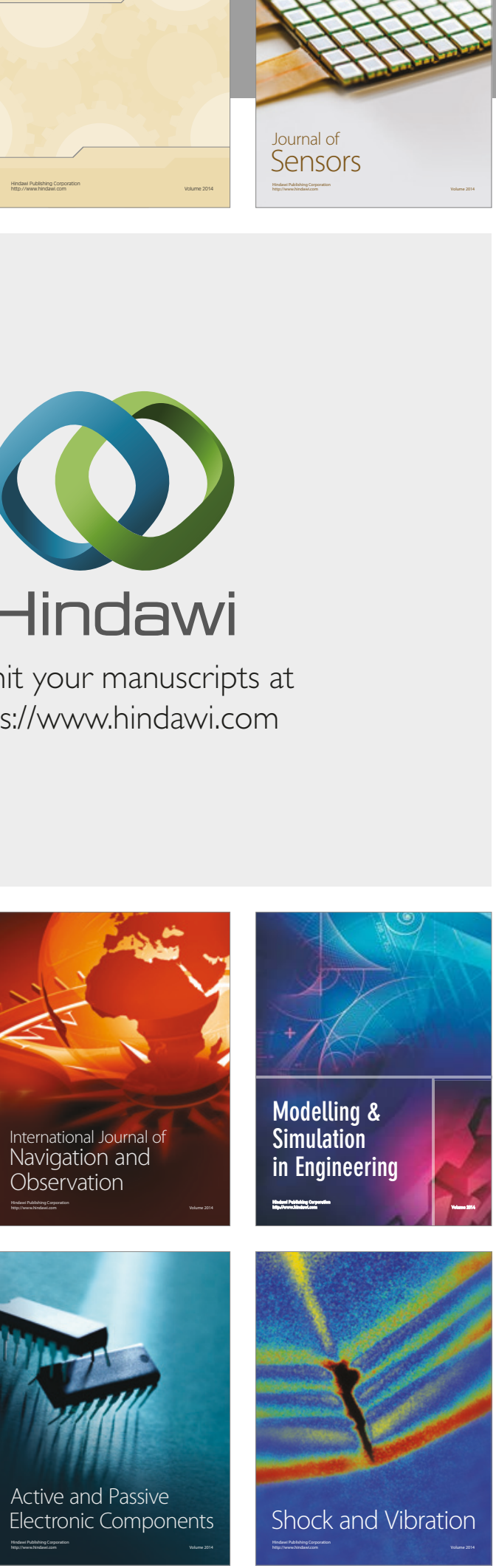
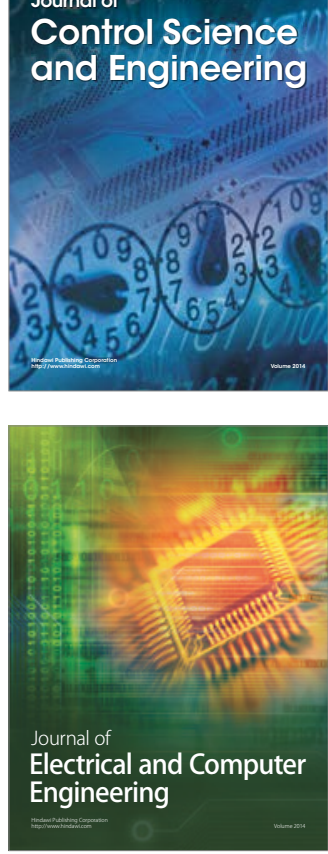

Distributed

Journal of

Control Science

and Engineering
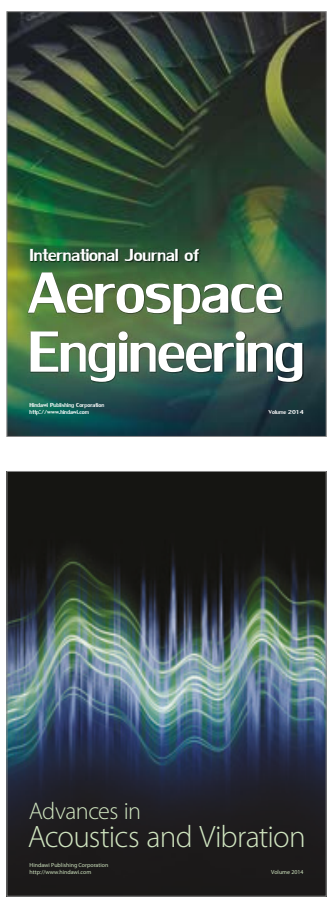

Sensor Networks 